\title{
Intraosseous synovial sarcoma of the proximal femur: Case report
}

\author{
M Zulkarnaen, MPath, KL Pan*, FRCS, PS Shanmugam*, MD, ZA Ibrahim, MPath, WH Chan*, MS Orth \\ Department of Pathology, Universiti Malaysia Sarawak, Kuching, Sarawak \\ *Department of Orthopaedics, Universiti Malaysia Sarawak, Kuching, Sarawak
}

\begin{abstract}
Synovial sarcoma is primarily a soft tissue malignancy that most often affects adolescents and young adults. It very rarely presents as a primary bone tumour and has only been reported in nine other cases to date. We report a case of primary synovial sarcoma arising from the proximal femur in a 57-year-old man.
\end{abstract}

Key Words:

Synovial sarcoma, primary bone tumour

\section{INTRODUCTION}

Synovial sarcoma forms a distinct clinical and morphological malignant soft tissue tumour that occurs primarily in the para-articular regions of the extremities. This tumour has a propensity to arise in the vicinity of large joints, especially around the knee region, although occurrence at other sites such as the head and neck, the trunk and abdomen has also been reported ${ }^{1}$. Synovial sarcoma most often affects adolescents and young adults. The underlying bone tends to be uninvolved, but $15-20 \%$ of the cases show a periosteal reaction, superficial bone erosion or invasion. Rarely, massive bone destruction is caused by poorly differentiated synovial sarcomas of long duration and large size ${ }^{1}$. Synovial sarcoma very rarely presents as a primary bone tumour and to the best of our knowledge, only nine other cases have been reported to date. We report a rare case of primary synovial sarcoma arising from the proximal femur.

\section{CASE REPORT}

A 57-year-old man presented to Miri Hospital with a one week history of right thigh pain and swelling after allegedly fallen down during work. He had difficulty ambulating independently due to the pain. Initial radiographs suggested a pathological fracture of the right subtrochanteric femur and the patient was referred to Sarawak General Hospital in June 2010 for further investigation. Magnetic resonance imaging (MRI) showed an expansile lesion at the proximal femur in the intertrochanteric region (Figure 1). Computed tomography (CT) scan of the chest was negative for metastatic disease, and a bone scan showed increased vascularity and an increased soft tissue uptake. An incisional biopsy of the lesion was performed with results indicative of biphasic synovial sarcoma.

The patient subsequently underwent two cycles of chemotherapy. After completion of the two cycles of chemotherapy, the patient underwent a wide excision of the tumour at the proximal part of the right femur that also included the involved soft tissue; this was followed by reconstruction with bipolar proximal femoral tumour prosthesis. Intraoperatively, there was a fracture at the subtrochanteric region with tumour extension into the soft tissue. The tumour was highly vascular with multiple feeding vessels.

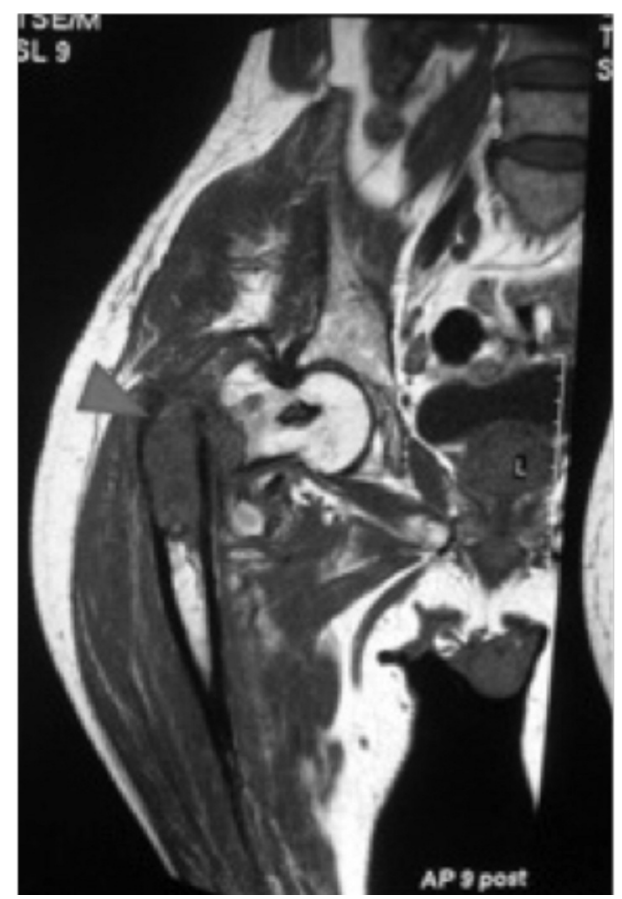

Fig. 1: Magnetic resonance imaging (MRI) showing an intraosseous lesion (arrow) at the proximal femur with an intertrochanteric fracture. 

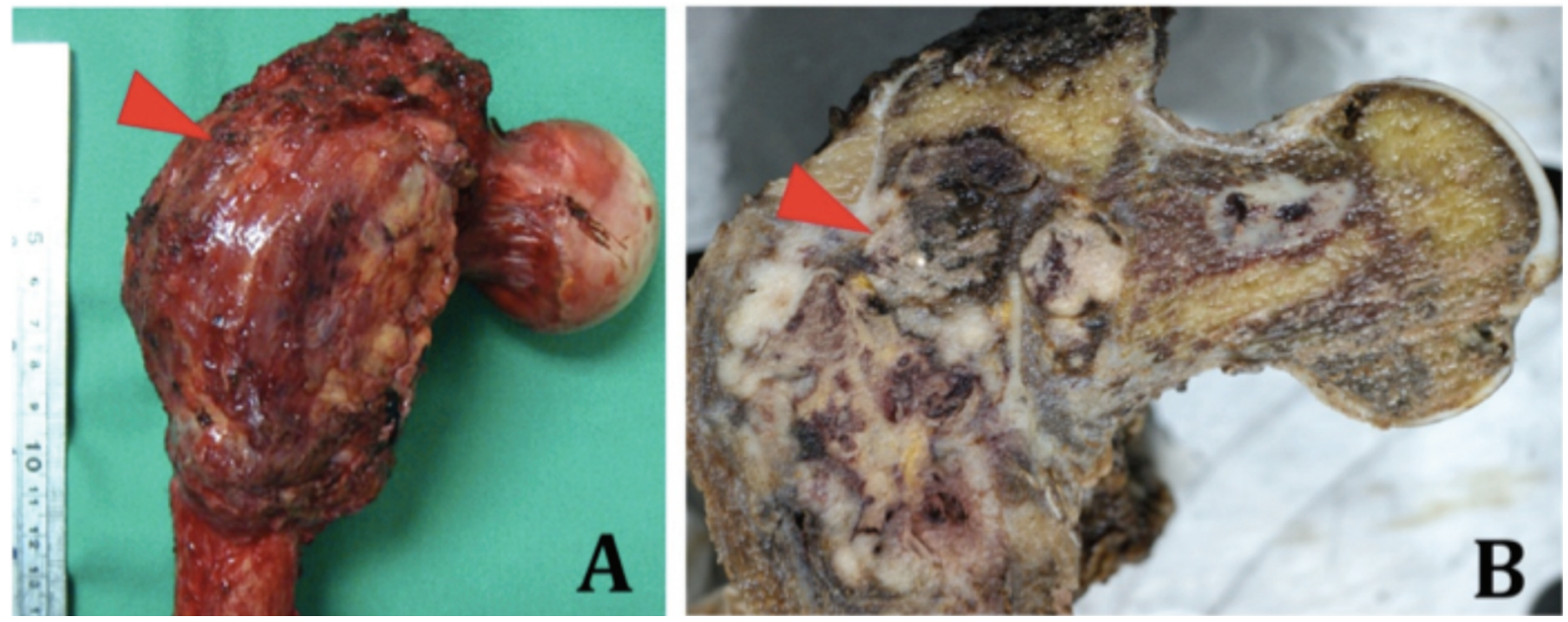

Fig. 2a: Gross specimen of the proximal femur with a normal looking muscle cuff surrounding the tumour and fracture site (red arrow). The distal femur shaft was cut at $3 \mathrm{~cm}$ distal to the tumour margin based on pre-chemotherapy MRI measurement.

Fig. 2b: Cut section of the proximal femur showing the fleshy grey, haemorrhagic tumour (red arrow) involving the metaphysis and diaphysis. Note that there is minimal involvement of the surrounding soft tissue.

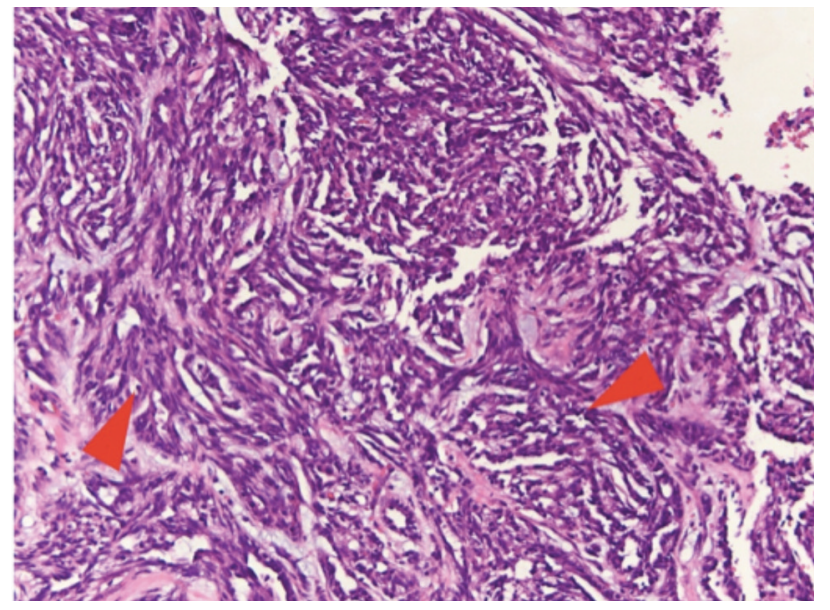

Fig. 3: Histological image showing glandular formations (red arrow) which is positive for pan-Cytokeratin, Epithelial Membrane Antigen (EMA) and Cytokeratin 7 (CK 7) antibodies. Other areas show predominantly spindled malignant cells.

Grossly, the tumour was fleshy grey and haemorrhagic involving the metaphysis and diaphysis of the proximal femur (Figure $2 \mathrm{a}$ and $2 \mathrm{~b}$ ). The tumour measured $6.5 \mathrm{x} 6.0 \mathrm{x}$ $6.0 \mathrm{~cm}$ and broke through the outer cortex into the surrounding soft tissue. Histologically, the tumour showed characteristic features of biphasic synovial sarcoma, containing two distinct predominantly consisting of spindled malignant cells and focal areas of glandular formations (Figure 3). No poorly differentiated areas were noted in any histological sections. Immunohistochemical studies showed that neoplastic cells were positive for vimentin, cytokeratin 7, epithelial membrane antigen (EMA) and pan-cytokeratin. The patient underwent another three cycles of chemotherapy post-operatively. Although a 4th cycle of chemotherapy was planned, this was not performed as his chest radiograph showed a right lower lobe lesion, suspicious for metastases. At this point, the patient requested to be discharged from our care, and he subsequently succumbed to the disease.

\section{DISCUSSION}

Synovial sarcoma is a relatively common malignant tumour representing $5-10 \%$ of all soft tissue sarcomas ${ }^{1}$. Although initially believed to be of synovial cell origin, the term is somewhat misleading as this type of tumour is also encountered in areas with no apparent relation to synovial structures. Many arise in the vicinity of large joints, especially around the knee region but occurrence at other sites such as the head and neck, the trunk and abdomen has also been reported ${ }^{2}$. In the present case, the tumour was considered to be of uncertain histogenesis because there is no precise normal tissue counterpart. While $20 \%$ of synovial sarcomas show invasion of local bone, it is extremely rare for it to present as a primary bone tumour.

Since the first report of synovial sarcoma in the lateral upper tibia back in 1997, there have been only nine reported cases of synovial sarcoma arising from the bone ${ }^{3}$. Other osseous sites reported include the sacrum, distal tibia, elbow, mandible, sternum and proximal tibia ${ }^{4}$. To our knowledge, this is the first reported case of primary synovial sarcoma in the femur. We believe this case represents a primary bone tumour because the bony involvement of the tumour far exceeds the soft tissue involvement. Furthermore, no other primary soft tissue tumour was identified to suggest the lesion represented bone metastasis. The clinical presentation involving bone fracture also favoured bone pathology rather than a soft tissue primary site, which was supported by the radiological findings. 
Even though synovial sarcoma is most prevalent in adolescents and young adults, it may affect patients in a wide age range from birth through the eighth decades ${ }^{2}$. Males are affected more than females in both the soft tissue primary and the reported osseous primary site. Pathologic diagnosis of synovial sarcoma is based on histologic exam with the aid of immunohistochemical staining. In this case, the presence of biphasic epithelial and stromal malignant cells coupled with the positive finding for cytokeratin 7, epithelial membrane antigen (EMA) and pan-cytokeratin were sufficient to confirm the diagnosis. The diagnosis may however be difficult in the other subtypes and cytogenetic or molecular genetics techniques have been used to confirm the $\operatorname{diagnosis}^{3-5}$.

The treatment of choice is a wide excision of the tumour with an oncological margin of normal tissue round the tumour. Therapeutically, synovial sarcoma is a chemosensitive sarcoma. Favourable prognostic characteristics for synovial sarcoma includes tumour presentation in the distal extremities, patient's age less than 25-years-old, tumour size of less than $5 \mathrm{~cm}$, absence of poorly differentiated areas and presence of extensive calcification ${ }^{1}$. The primary tumor in this 57-year-old patient, being larger than $5 \mathrm{~cm}$, had an increased risk of metastasis and unfavourable prognosis. Metastatic lesions have been reported in about half of the cases and are most commonly seen in the lungs. The five year survival rate is reported to be in the range of $26-76 \%$ and 10 year survival rates ranges from $20-63 \%$.

\section{ACKNOWLEDGMENTS}

The authors thank Dr. Noraini Mohd Dusa and Angela Chia for their contributions to this paper. 


\section{REFERENCES}

1. Weiss SW, Goldblum JR, Enzinger FM. Enzinger and Weiss' soft tissue tumors. 5th ed. Philadelphia, PA: Mosby Elsevier, 2008.

2. Fletcher CDM, Unni KK, Mertens F, World Health Organization., International Agency for Research on Cancer. Pathology and genetics of tumours of soft tissue and bone. Lyon: IARC Press, 2002.

3. Cohen IJ, Issakov J, Avigad S, Stark B, Meller I, Zaizov R, et al. Synovial sarcoma of bone delineated by spectral karyotyping. Lancet. 1997; 350(9092): 1679-80.

4. Beck S NG, Raskin KA and Schwab JH. Intraosseous Synovial Sarcoma of the Proximal Tibia. International Journal of Surgical Oncology. 2011; 2011: 1-5.

5. Hiraga H, Nojima T, Isu K, Yamashiro K, Yamawaki S, Nagashima K. Histological and molecular evidence of synovial sarcoma of bone. A case report. J Bone Joint Surg [AM] 1999; 81(4): 558-63. 